\title{
Identification of dysregulated miRNAs and their regulatory signature in glioma patients using the partial least squares method
}

\author{
JIAJUN SHOU, SHIXIN GU and WENTAO GU \\ Department of Neurosurgery, Huashan Hospital, Fudan University, Shanghai 200040, P.R. China
}

Received March 19, 2014; Accepted September 25, 2014

DOI: $10.3892 /$ etm.2014.2041

\begin{abstract}
Using microarray data, the present study identified differentially expressed microRNAs (miRNAs) and evaluated their regulatory characteristics in high-grade glioma patients, with the aim to further the understanding into the underlying etiology of the condition. Previously, studies have generally implemented regression or variance analysis, which ignores various background biological factors. However, in the present study, analysis was performed with microarray data collected from the Gene Expression Omnibus database using a partial least squares-based method, which is more sensitive in handling microarray data. Among the six identified differentially expressed miRNAs, hsa-miR-21 and hsa-miR-612 have been previously reported to be associated with glioma. In addition, the remaining miRNAs, hsa-miR-4680, hsa-miR-1908, hsa-miR-4656 and hsa-miR-4467, may also contribute to glioma progression since they are all associated with the tumorigenesis of other types of cancer. Moreover, the expression levels of hsa-miR-1908, hsa-miR-4656 and hsa-miR-4680 have been identified to significantly correlate with the survival rate. Enrichment analysis of the dysregulated target genes revealed that the selected miRNAs primarily affect biological processes in the nervous system and the protein phosphorylation process. Therefore, the results may offer a new understanding into the pathogenesis of high-grade glioma.
\end{abstract}

\section{Introduction}

Gliomas are a type of tumor that arise from glial cells, constituting $\sim 30 \%$ of brain and central nervous system tumors and $80 \%$ of malignant brain tumors (1). To date, the prognosis of high-grade (stage III-IV) glioma cases remains poor. microRNAs (miRNAs) are small non-coding RNAs,

Correspondence to: Dr Wentao Gu, Department of Neurosurgery, Huashan Hospital, Fudan University, 12 Wulumuqi Middle Road, Shanghai 200040, P.R. China

E-mail: wentao_gu@126.com

Key words: glioma, partial least squares, microRNA, target gene, expression profile, survival comprised of 18-22 nucleotides, that regulate gene expression and control various biological processes (2) through binding to the 3'-untranslated region of mRNAs. Subsequently, the stability and translation of the target mRNAs are affected. miRNAs have recently been identified as crucial factors in tumorigenesis, but also tumor aggressiveness $(3,4)$. In addition, miRNAs are hypothesized to be widely dysregulated in cancer; thus, may serve as potential markers for cancer diagnosis, prognosis and treatment (5). miRNAs have also been reported to be associated with the pathogenesis and anticancer treatment sensitivity of gliomas (6). Therefore, the identification of differentially expressed miRNAs and the determination of their regulatory role in high-grade glioma patients may help to further the understanding into the underlying etiology.

High-throughput microarray technology facilitates the investigation of characteristics that underlie the progression of cancer. Several studies have investigated the miRNA expression signature in glioma patients $(7,8)$. However, these studies have generally implemented regression or variance analysis, which is unable to evaluate unaccounted array specific factors. Partial least squares (PLS) analysis has been demonstrated to be effective and more sensitive in handling microarray data $(9,10)$. A previous study used this method on other complex diseases and demonstrated the feasibility (11). Thus, identifying the miRNA expression signature of glioma patients using this method may conduce to a new understanding of the disease progression.

In the present study, to identify the differentially expressed miRNAs and determine their regulatory characteristics in high-grade glioma patients, PLS analysis was performed using microarray data collected from the Gene Expression Omnibus (GEO) database. Survival analysis of the selected miRNAs was conducted to analyze the effect of these miRNAs on the prognosis of the patients. In addition, dysregulated miRNAs and target mRNAs were used to construct a regulatory network. Pathway and Gene Ontology (GO) enrichment analysis of the dysregulated target genes was also used to evaluate the biological effects of the differentially expressed miRNAs.

\section{Materials and methods}

Microarray data. An expression profile data set from the GEO database (GSE4412) was used, which included the transcription profile of 24 grade III and 50 grade IV glioma patients. All the RNA samples were extracted from fresh frozen tumor tissues 

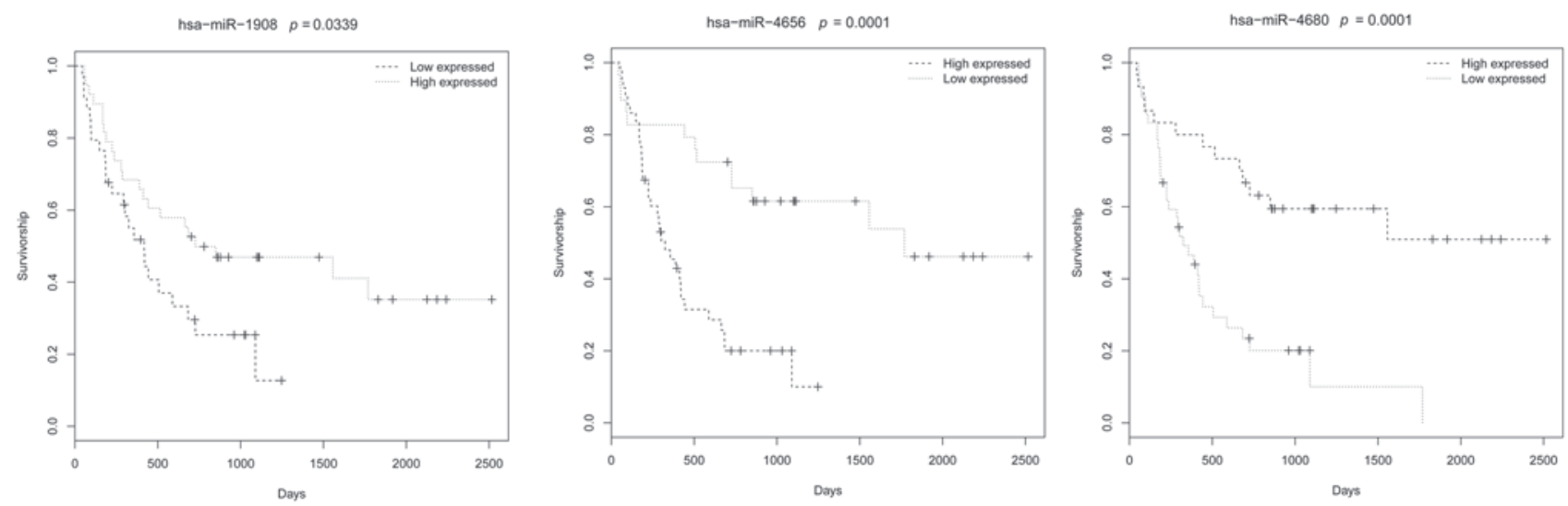

Figure 1. Survival curve of the three miRNAs that were identified to be associated with the survival rate of the patients. miRNAs, microRNAs.

that had been collected during surgical treatment. The data set was based on two Affymetrix platforms (GPL96 and GPL97).

Identification of differentially expressed miRNAs. Raw data of all the samples, including CEL and simple omnibus format in text-formatted files, were obtained from the GEO database. Following quality control, normalization of the raw intensity values was conducted using a Robust Multi-array Analysis (RMA) (12) procedure. Neutralization of background noise effects and processing artifacts was performed with model-based background correction, and expression values of all the probes were aligned to a common scale using quantile normalization. The $\log _{2}$-transformed RMA values of all the probes were subsequently used in PLS analysis to estimate their effects on the grade III and IV samples. Briefly, PLS latent variables were initially calculated with the non-linear iterative partial least squares algorithm (13); subsequently, the effects of the probe expression values on the disease status were estimated using variable importance in the projection (VIP) (14). Finally, the false discovery rate (FDR) of each probe was calculated based on the empirical distribution of the PLS-based VIP scores, generated by a permutation procedure $(n=10,000)$. miRNAs with a FDR of $<0.01$ were considered to be significant differentially expressed miRNAs. The aforementioned procedures were performed with $\mathrm{R}$ software (version 3.0.0; http://www.r-project.org/), including BioConductor (http://www.bioconductor.org/.), limma packages (3.12.1) and libraries (15).

Survival analysis. To investigate the contribution of the differentially expressed miRNAs to the survival time following surgery, survival analysis was performed. For each miRNA, the samples were separated into two classes using a K-mean algorithm based on the expression value. Using the survival time or last follow-up time of the patients, the log-rank test was used to investigate whether the two classes were significantly different from each other. $\mathrm{P}<0.05$ was considered to indicate a statistically significant difference; thus, miRNAs with these values were found to be significantly associated with the survival rate.

Target gene prediction and network construction. Target gene prediction for the differentially expressed miRNAs was performed using currently available methods, including
Table I. Differentially expressed miRNAs.

\begin{tabular}{lcc}
\hline miRNAs & Fold change & FDR \\
\hline hsa-miR-4680 & -0.7602 & 0.0014 \\
hsa-miR-1908 & -0.6327 & 0.0074 \\
hsa-miR-4656 & 0.4312 & 0.0127 \\
hsa-miR-4467 & 0.3912 & 0.0164 \\
hsa-miR-612 & 0.9768 & 0.0220 \\
hsa-miR-21 & 1.4927 & 0.0273 \\
\hline
\end{tabular}

FDR, false discovery rate; miRNAs, microRNAs.

microT (16), miRanda (17), mircode (18) and TargetScan (19). Target genes supported by at least two methods were used in further analysis. Differentially expressed genes were selected using the same procedure as for the detection of differentially expressed miRNAs. Target genes that were identified to be differentially expressed in the grade III and IV samples were subsequently used to construct a regulatory network of selected miRNAs using Cytoscape software (V 2.8.3, http://www.cytoscape.org/) (20).

Enrichment analysis. To estimate the biological effects of the differentially expressed miRNAs, enrichment analysis was performed for the differentially expressed target genes. Target genes were firstly annotated based on the Kyoto Encyclopedia of Genes and Genomes (KEGG) pathways (http://www.genome.jp/kegg/) and GO database. A hypergeometric distribution test was then used to identify the pathways and GO items enriched with dysregulated miRNA target genes.

\section{Results}

Identification of differentially expressed miRNAs. As shown in Table I, six miRNAs were identified to be differentially expressed in the grade III and IV glioma patients, including two downregulated miRNAs (hsa-miR-4680 and hsa-miR-1908) and four overexpressed miRNAs (hsa-miR-4656, hsa-miR-4467, hsa-miR-612 and hsa-miR-21). 
Table II. Pathways and GO items enriched with differentially expressed genes.

\begin{tabular}{lll}
\hline Parameter & \multicolumn{1}{c}{ Description } & \multicolumn{1}{c}{ Class } \\
\hline $\begin{array}{l}\text { KEGG pathway } \\
\text { hsa04723 }\end{array}$ & Retrograde endocannabinoid signaling & Pervous system \\
hsa04728 & Dopaminergic synapse & Nervous system \\
GO item & & \\
GO:0004722 & Protein serine/threonine phosphatase activity & Function \\
GO:0007258 & JUN phosphorylation & Process \\
GO:0006470 & Protein dephosphorylation & Process \\
GO:0045211 & Postsynaptic membrane & Component
\end{tabular}

KEGG, Kyoto Encyclopedia of Genes and Genomes; GO, Gene Ontology.

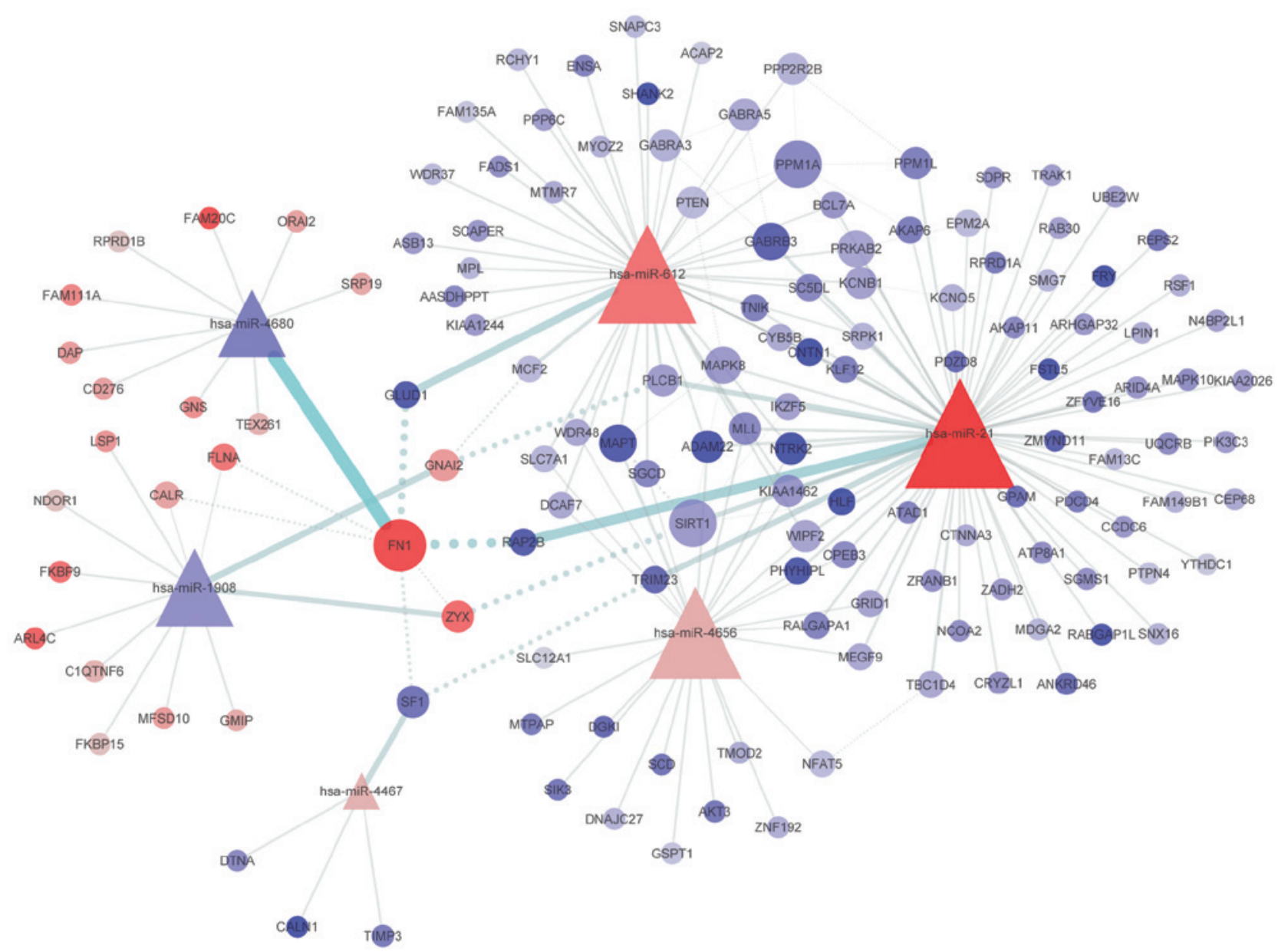

Figure 2. Regulatory network constructed with dysregulated miRNAs and target genes. Interactions between target genes are shown with a dotted line. Items in red represent overexpressed molecules in grade IV patients, while those in blue represent downregulated molecules in grade IV samples. Molecules with a higher number of interactions are shown in a bigger size. miRNAs, microRNAs.

Survival analysis. Survival analysis of these miRNAs revealed that three miRNAs, hsa-miR-1908, hsa-miR-4656 and hsa-miR-4680, were significantly associated with the survival rate of the patients, with P-values of 0.0339, 0.0001 and 0.0001, respectively (Fig. 1).

Target gene prediction and network construction. Target gene prediction and PLS analysis identified 139 differentially expressed target genes, and a network was constructed using these miRNAs and target genes (Fig. 2). The degree of each node was defined as the number of its interactions in the network. Nodes with higher degrees are shown as a bigger size. Among the six miRNAs, hsa-miR-21 was found to have a higher number of interactions compared with the other miRNAs. In the three overexpressed miRNAs, hsa-miR-21, hsa-miR-612 and hsa-miR-4656, a number of the target genes 
were found to be shared. Among the target genes, FN1 was detected to have the highest degree.

Enrichment analysis. Enrichment analysis of the differentially expressed target genes identified two KEGG pathways and four GO items over-represented with dysregulated target genes (Table II). The two KEGG pathways were retrograde endocannabinoid signaling and dopaminergic synapse, which are both involved in the nervous system. In addition, one of the GO items, the postsynaptic membrane (GO:0045211), is also associated with the nervous system. The remaining three GO items were shown to be associated with the protein phosphorylation process, including protein serine/threonine phosphatase activity (GO:0004722), JUN phosphorylation (GO:0007258) and protein dephosphorylation (GO:0006470).

\section{Discussion}

To identify differentially expressed miRNAs and determine their regulatory characteristics in high-grade glioma patients, PLS analysis was performed. In total, six miRNAs were identified to be dysregulated. Among them, hsa-miR-21 has been previously reported to exhibit a significant correlation with tumor grade and prognosis $(21,22)$. In addition, the expression of hsa-miR-612 has been reported to be associated with magnetic resonance imaging features of glioblastoma multiforme (23). The results of the present study further confirmed the involvement of the two miRNAs in the progression of glioma. In addition, one of the target genes of miR-612 is PTEN. PTEN is a tumor suppressor that negatively regulates the protein kinase $\mathrm{B} / \mathrm{Akt}$-dependent cell survival pathway (24). Therefore, depression of PTEN may impact its negative regulation of tumor cell survival and contribute to the deterioration of the disease. None of the remaining four miRNAs have been previously reported to be associated with glioma. However, hsa-miR-1908 has been reported to be associated with the metastasis or tumorigenesis of other types of tumor, including chordomas (25), hepatoma (26) and melanoma (27). In addition, hsa-miR-4680, hsa-miR-4656 and hsa-miR-4467 have been hypothesized to be associated with breast cancer (28). For miR-4680, one of its target genes is $F N 1$, which is a hub gene that was found to have the highest degree among the target genes. The protein encoded by this gene is fibronectin, which is involved in cell adhesion and migration processes. A previous study reported a significant correlation between this gene and malignant glioma (29), indicating the potential regulatory mechanism of miR-4680 in the development of glioma. Survival analysis also revealed that the expression levels of hsa-miR-1908, hsa-miR-4656 and hsa-miR-4680 were significantly associated with the survival rate of the patients (Fig. 1). Moreover, the constructed network of dysregulated miRNAs and target genes revealed that hsa-miR-21, hsa-miR-612 and hsa-miR-4656 share a number of target genes, indicating that they may affect similar biological processes. Thus, further investigation of these miRNAs is warranted.

Pathway enrichment analysis of the dysregulated target genes revealed that the differentially expressed miRNAs primarily affect pathways in the nervous system, including retrograde endocannabinoid signaling and the dopaminergic synapse. GO analysis additionally revealed the over-representation of dysregulated target genes in the protein phosphorylation process. The GO:0004722 item of protein serine/threonine phosphatase activity exhibited the most significant enrichment. A previous study reported the alteration of striatal dopaminergic function in glioma development (30). Furthermore, dysregulation of serine/threonine phosphatase calcineurin has also been reported in grade IV astrocytoma tumor tissue (31). The enrichment analysis results further confirmed the effects of the dysregulated miRNAs.

In summary, using an expression profile from the GEO database, PLS-based analysis was performed to identify differentially expressed miRNAs and evaluate their regulatory characteristics in high-grade glioma patients. In total, six miRNAs were identified to be dysregulated, including three miRNAs significantly associated with the survival rate of the patients. Enrichment analysis of the dysregulated target genes revealed that the differentially expressed miRNAs predominantly affected biological processes associated with the nervous system and the protein phosphorylation process. Thus, the results may offer a new understanding into the pathogenesis of high-grade glioma.

\section{References}

1. Goodenberger ML and Jenkins RB: Genetics of adult glioma. Cancer Genetics 205: 613-621, 2012.

2. Bartel DP: MicroRNAs: genomics, biogenesis, mechanism, and function. Cell 116: 281-297, 2004.

3. Calin GA and Croce CM: MicroRNA signatures in human cancers. Nat Rev Cancer 6: 857-866, 2006.

4. Waldman SA and Terzic A: Translating microRNA discovery into clinical biomarkers in cancer. JAMA 297: 1923-1925, 2007.

5. Garzon R, Marcucci G and Croce CM: Targeting microRNAs in cancer: rationale, strategies and challenges. Nat Rev Drug Discov 9: 775-789, 2010.

6. Li M, Li J, Liu L, Li W, Yang Y and Yuan J: MicroRNA in Human Glioma. Cancers (Basel) 5: 1306-1331, 2013.

7. Visani M, de Biase D, Marucci G, et al: Definition of miRNAs expression profile in glioblastoma samples: the relevance of non-neoplastic brain reference. PloS One 8: e55314, 2013.

8. Yang C, Wang C, Chen X, et al: Identification of seven serum microRNAs from a genome-wide serum microRNA expression profile as potential noninvasive biomarkers for malignant astrocytomas. Int J Cancer 132: 116-127, 2013.

9. Chakraborty S, Datta S and Datta S: Surrogate variable analysis using partial least squares (SVA-PLS) in gene expression studies. Bioinformatics 28: 799-806, 2012.

10. Ji GL, Yang ZJ and You WJ: PLS-based gene selection and identification of tumor-specific genes. IEEE Trans Syst Man Cybern C Appl Rev 41: 830-841, 2011.

11. Gao QG, Li ZM and Wu KQ: Partial least squares based analysis of pathways in recurrent breast cancer. Eur Rev Med Pharmacol Sci 17: 2159-2165, 2013.

12. Irizarry RA, Hobbs B, Collin F, et al: Exploration, normalization, and summaries of high density oligonucleotide array probe level data. Biostatistics 4: 249-264, 2003.

13. Martins JPA, Teófilo RF and Ferreira MMC: Computational performance and cross-validation error precision of five PLS algorithms using designed and real data sets. J Chemom 24: 320-332, 2010.

14. Gosselin R, Rodrigue D and Duchesne C: A bootstrap-VIP approach for selecting wavelength intervals in spectral imaging applications. Chemometr Intell Lab Syst 100: 12-21, 2010.

15. Smyth GK, Michaud J and Scott HS: Use of within-array replicate spots for assessing differential expression in microarray experiments. Bioinformatics 21: 2067-2075, 2005.

16. Maragkakis M, Alexiou P, Papadopoulos GL, et al: Accurate microRNA target prediction correlates with protein repression levels. BMC Bioinformatics 10: 295, 2009. 
17. John B, Enright AJ, Aravin A, Tuschl T, Sander C and Marks DS Human MicroRNA targets. PLoS Biol 2: e363, 2004.

18. Jeggari A, Marks DS and Larsson E: miRcode: a map of putative microRNA target sites in the long non-coding transcriptome. Bioinformatics 28: 2062-2063, 2012.

19. Lewis BP, Shih IH, Jones-Rhoades MW, Bartel DP and Burge CB Prediction of mammalian microRNA targets. Cell 115: 787-798, 2003.

20. Shannon P, Markiel A, Ozier O, et al: Cytoscape: a software environment for integrated models of biomolecular interaction networks. Genome Res 13: 2498-2504, 2003.

21. Hermansen SK, Dahlrot RH, Nielsen BS, Hansen S and Kristensen BW: MiR-21 expression in the tumor cell compartment holds unfavorable prognostic value in gliomas. J Neurooncol 111: 71-81, 2013.

22. Wu L, Li G, Feng D, et al: MicroRNA-21 expression is associated with overall survival in patients with glioma. Diagn Pathol 8: 200, 2013.

23. Li WB, Chen HY, Zhang W, et al: Relationship between magnetic resonance imaging features and miRNA gene expression in patients with glioblastoma multiforme. Chin Med J (Engl) 126: 2881-2885, 2013

24. Stambolic V, Suzuki A, de Pompa JL, et al: Negative regulation of PKB/Akt-dependent cell survival by the tumor suppressor PTEN. Cell 95: 29-39, 1998.
25. Long C, Jiang L, Wei F, et al: Integrated miRNA-mRNA analysis revealing the potential roles of miRNAs in chordomas. PloS One 8: e66676, 2013

26. Jin JC, Jin XL, Zhang X, Piao YS and Liu SP: Effect of OSW-1 on microRNA expression profiles of hepatoma cells and functions of novel microRNAs. Mol Med Rep 7: 1831-1837, 2013.

27. Pencheva N, Tran H, Buss C, et al: Convergent multi-miRNA targeting of ApoE drives LRP1/LRP8-dependent melanoma metastasis and angiogenesis. Cell 151: 1068-1082, 2012.

28. Persson H, Kvist A, Rego N, et al: Identification of new microRNAs in paired normal and tumor breast tissue suggests a dual role for the ERBB2/Her2 gene. Cancer Res 71: 78-86, 2011.

29. Wei KC, Huang CY, Chen PY, et al: Evaluation of the prognostic value of CD44 in glioblastoma multiforme. Anticancer Res 30: 253-259, 2010.

30. Lonjon M, Quentien MH, Risso JJ, et al: Alteration of striatal dopaminergic function induced by glioma development: a microdialysis and immunohistological study in the rat striatum. Neuroscience Lett 354: 131-134, 2004.

31. Brun M, Glubrecht DD, Baksh S and Godbout R: Calcineurin regulates nuclear factor I dephosphorylation and activity in malignant glioma cell lines. J Biol Chem 288: 24104-24115, 2013. 